\title{
Enhancement of the nonlinear response in Mach-Zehnder-Fano interferometer
}

\author{
Yi Xu ${ }^{1,2}$ Andrey E. Miroshnichenko ${ }^{1}$ \\ 1.Nonlinear Physics Centre and Centre for Ultra-high bandwidth Devices for Optical Systems (CUDOS), Australian National University, \\ Canberra ACT 0200, Australia \\ 2. Laboratory of Photonic Information Technology, School for Information and Optoelectronic Science and Engineering, South China Normal \\ University, Guangzhou 510006, P.R. China \\ Presenting author e-mail address: yix124@physics.anu.edu.au
}

\begin{abstract}
We demonstrate the enhancement of nonlinear response and bistable switching in the Mach-Zehnder interferometer with side coupled nonlinear defects. Such system is known to exhibit high-Q nonlinear Fano resonances. Based on the introduced figure of merit the enhancement up to two orders of magnitude can be achieved compared to standard geometries. We applied these results for photonic crystal platform which allow for enhanced optical bistable operation.
\end{abstract}

\section{Introduction}

The Fano resonances manifest themselves as sharp and asymmetric profiles in transmission or absorption lineshapes [1]. Recently it became a very attractive physical phenomenon for engineering the nonlinear response at the nanoscale [2]. In simplest case, the Fano resonance can be attributed to the interaction of a discrete state with a continuum. The more interesting situation is the interaction of many Fano defects with several continua. This situation was realized in Mach-Zehnder-Fano interferometer (MZFI), which exhibits very distinct properties compared with conventional Mach-Zehnder interferometer (MZI) [3]. In this work, we demonstrate that the nonlinear switching can be drastically enhancement in MZFI geometries. As its the small defect volume and unique resonance properties which can't be found in the macroscopic MZI [4,5], the counterpart of the ring-resonatorcoupled MZI in the nanoscale, i.e. MZFI constructed by photonic crystals [3] appears to be more promising.

\section{Model}

We consider a generic discrete model which has demonstrated a well-suited treatment to obtain the nonlinear response of various systems, including photonic crystal [2]. The sketch maps of two particular geometries are shown by Fig. 1 (a) and (b). By using the modified Fano-Anderson model, the equations of motion describing the system of Fig. 1 (b) are read as coupled discrete nonlinear equations:

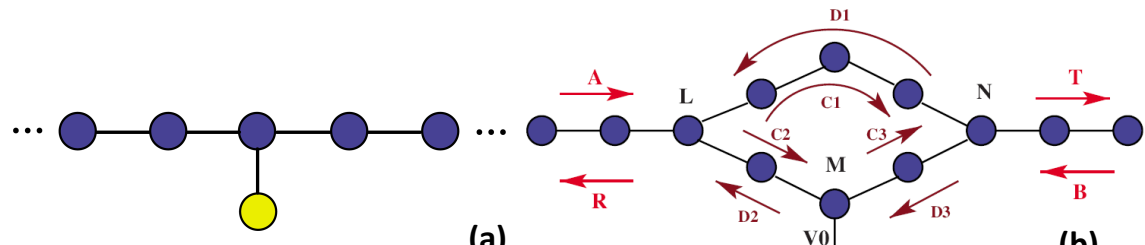

(a)

v0

(b)
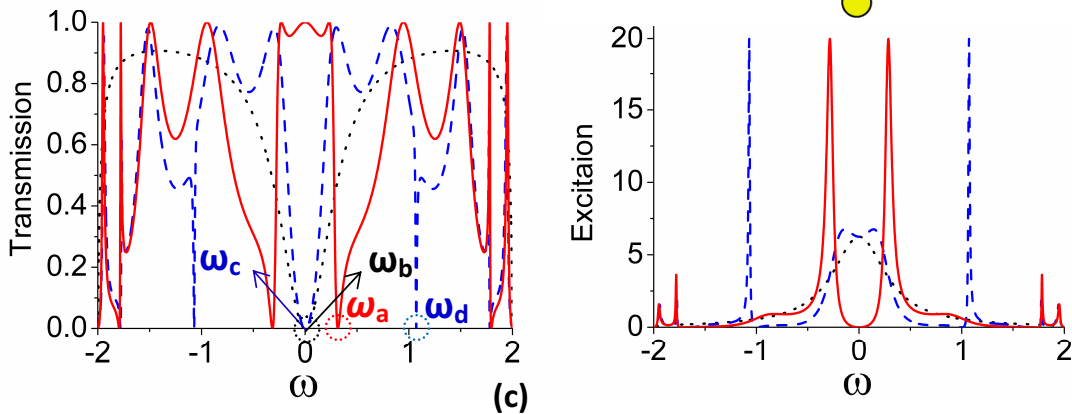

(d)

Fig. 1. (a) and (b) are the generic discrete model for the system exhibiting Fano resonance. (c) and (d) are the stationary transmission results and excitation in the Fano defect, respectively. Such systems support different types of resonances, including, single Fano defect (black dotted line), MZFI with $\mathrm{L}=1, \mathrm{~N}=-7, \mathrm{M}=3$ (blue dashed line) and $\mathrm{MZFI}$ with $\mathrm{L}=1, \mathrm{~N}=-7, \mathrm{M}=4$ (red solid line). In both case, $\mathrm{E}_{\mathrm{d}}=0$ and $\mathrm{V}=0.8$. 


$$
\begin{aligned}
& i \dot{\psi}_{n}=\sum_{k} C \psi_{k}+\delta_{n, M} V \varphi_{d} \\
& i \dot{\varphi}_{d}=E_{d} \varphi_{d}+\lambda\left|\varphi_{d}\right|^{2} \varphi_{d}+V \psi_{M}
\end{aligned}
$$

Where $\psi_{n}$ represents the linear chains with complex field amplitude, $\varphi_{d}$ stands for the Fano defect, $M$ gives the location of the Fano defect in the arm, $\mathrm{C}$ is the nearest-neighbor coupling coefficient, $k$ is the total numbers of the neighbor sites in the chain, $\lambda$ is the Kerr nonlinear parameter, $\mathrm{V}$ is the coupling strength between the chain and the Fano defect and $E_{d}$ is the eigenfrequency of the Fano defect, respectively.

The stationary solutions of this system can be found by substituting $\psi_{n}(t)=A_{n} e^{-i \omega t}$ and $\varphi_{d}(t)=B_{d} e^{-i \omega t}$ into (1). Combining with the scattering matrices of the Fano defect and the junctions [6], the linear response of the system can be obtained by solving a linear equation set. While the Newton-Raphson method is used to resolve the nonlinear response of the system which relies on the complex nonlinear equation set.

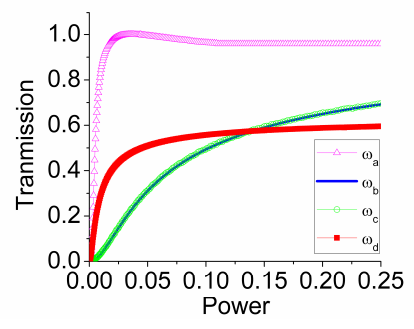

Fig. 2. Nonlinear switching response of different resonances of the stationary case. Pink open triangles stand for the resonance marked $\omega_{\mathrm{a}}$ in Fig. 1 (c), red close squares present the resonance of $\omega_{\mathrm{d}}$ while green open circles and blue solid lines represent the cases of eigen-Fano resonance of the Fano defect coupling to a chain $\left(\right.$ marked $\left.\omega_{\mathrm{b}}\right)$ and the MZI (marked $\left.\omega_{\mathrm{c}}\right)$, respectively.

\section{Enhancement of the nonlinear response by the Fano-Feshbach resonance}

We analyse spectral properties of MZFI configuration with different coupling symmetries of nonlinear Fano defects. The linear spectrum is presented in Fig.1 (c) and (d). The single Fano resonance case [see Fig.1 (a)] is shown by dotted line as a reference. It can be seen from the figures that the excitation of the Fano defects varies for different coupling symmetries. When the Fano defect locates in the middle of the arm, the transmission in the band centre looks like a step function. At the same time, the resonance of the loop and the Fano-Feshbach resonance induced by the interaction of two Fano resonances have higher excitation than the eigen-resonance of the Fano defect because they are consisted of resonances generated by different mechanisms [7].

As the matter of fact, higher excitation in the nonlinear defect would facilitate the response of the system with the Kerr type nonlinearity. The nonlinearity would shift the maximum excitation frequency of the Fano defect and the total amount of the deviation in turn depends on the excitation of the Fano defect. At the same time, the nonlinear response of the Fano defect would give rise to a positive feedback for the scattering waves in the arm. The stationary nonlinear responses of the system at different kinds of resonances are shown in Fig. 2. We compare four kinds of resonances marked $\omega_{\mathrm{a}}-\omega_{\mathrm{d}}$ at Fig. 1 (c). In order to compare the switching power of the different frequencies, all of them start exactly at the total transmission dips. As can be seen from Fig. 2, the resonance with higher nonlinear defect excitation corresponds to faster increase of transmission with input power. We have to point out that the high excitation of resonance $\omega_{\mathrm{d}}$ is mediated by the highly excitation of the site coupled to the Fano defect and it is not the eigen-resonance of the Fano defect [7]. Furthermore, the transmission line shape of the resonance $\omega_{d}$ has more deviation from the perfect step function. Therefore, although resonances $\omega_{\mathrm{a}}$ and $\omega_{\mathrm{d}}$ have the same cavity excitation in the stationary case, $\omega_{\mathrm{a}}$ involving with the eigenfrequency of the Fano defect would be more sensitive to the input power. At the same time, the nonlinear response is greatly reduced by the sharp and asymmetric line shape of the resonance [8] compared to resonance $\omega_{b}$ and $\omega_{c}$, i.e. the maximum of the excitation is in between the transmission dip and tip of the resonance $\omega_{\mathrm{a}}$. By defining the figure of merit (FOM) as $T_{\max } / \mathrm{P}_{\mathrm{in}}$, where $\mathrm{T}_{\max }$ refers to the nearest transmission maximum and $\mathrm{P}_{\text {in }}$ represents the necessary input power to pull the response of the system up to $90 \%$ of the $\mathrm{T}_{\max }$, the FOM of the resonance $\omega_{\mathrm{a}}$, which describes both the enhanced transmission contrast in the linear case and the reduction of the switching power, can be enhanced more than 60 times comparing to the common 
Fano resonance $\omega_{b}$ and $\omega_{c}$. The raising properties of the resonance $\omega_{b}$ and $\omega_{c}$ are the same because they are originated from the eigen-properties of the Fano defect and are excited almost the same.

It had been pointed out that the nonlinear Fano resonance suffers from dynamical modulation instability [9]. Strictly speaking, modulation instability of the nonlinear Fano resonance needs time to grow and the raising time is related on the driven strength at certain frequency. As a result, suitable choosing of the temporal pulse's width and maximum amplitude, we still can access the bistable operation. Figure 3 demonstrates the transient response of the system with the excitation pulse

$$
I(t)=I_{0} \exp \left(-\frac{\left(t-T_{0}\right)^{2}}{w^{2}}\right) \exp (-i \omega t)
$$

And the parameters are outlined in the caption. Please note that the detuning of each resonance is $\Delta \omega=0.1$.
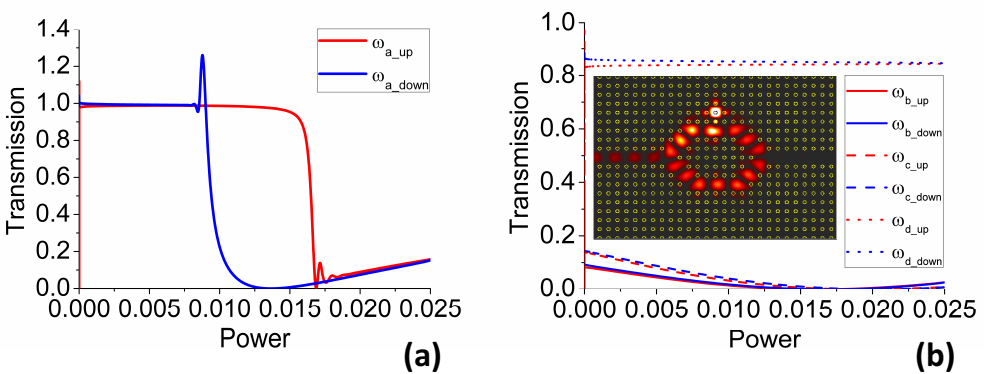

Fig. 3 Bistable operation of different kinds of resonances marked in Fig. 1 (c). Here, $\mathrm{T}_{0}=1.3 \times 10^{\wedge} 4, \mathrm{w}=5 \times 10^{\wedge} 3$ and $\mathrm{I}_{0}=0.025$. Red lines and the blue lines stand for the upward and downward operations.

The bistable operation of resonance $\omega_{\mathrm{a}}$ shown in Fig. 3(a) benefits from the sharp step function like linear transmission and the enhanced excitation in the nonlinear defect. The responses of other resonances at the same condition are shown in Fig. 3(b) for reference. Such detuning is not enough to initiate a bistable response for resonances $\omega_{b}$ and $\omega_{c}$, while the resonance $\omega_{d}$ does not exhibit well pronounced bistable effect for larger powers. Therefore, the capacity of enhanced nonlinear response is one of the fine properties of the MZFI which offers the unique opportunities to manipulate the nonlinear Fano resonances [7].

It should be pointed out that the result obtained here by using the discrete model is general and can be applied to other branches of discrete physical system. The MZFI created by photonic crystal circuit as an example is shown by the inset of Fig. 3 (b). The transient electric field distribution $(|\mathrm{E}|)$ at exactly the dynamic downward switching point (from on to off-state) is also outlined. Nearly perfect blocking of the input pulse at the right output port demonstrates the dynamic shutting down operation by a pulse.

\section{References}

[1] U. Fano, "Effects of Configuration Interaction on Intensities and Phase Shifts," Phys. Rev. 124, 1866 (1961).

[2] A. E. Miroshnichenko, S. Flach, and Yu. S. Kivshar, "Fano resonances in nanoscale structures,” Rev. Mod. Phys. 82, 2257 (2010).

[3] A. E. Miroshnichenko and Yu. S. Kivshar, "Mach-Zehnder-Fano interferometers,” Appl. Phys. Lett. 95, 121109 (2009).

[4] John E. Heebner and Robert W. Boyd, "Enhanced all-optical switching by use of a nonlinear fiber ring resonator, " Opt. Lett. 24, 847-849 (1999).

[5] Ying Lu, Jianquan Yao, Xifu Li, and Peng Wang, "Tunable asymmetrical Fano resonance and bistability in a microcavity-resonator-coupled Mach-Zehnder interferometer, ” Opt. Lett. 30, 3069-3071 (2005).

[6] A. E. Miroshnichenko, M. I. Molina, and Yu. S. Kivshar, "Localized modes and bistable scattering in nonlinear network junctions, " Phys. Rev. E 75, 046602 (2007).

[7] Yi Xu and A. E. Miroshnichenko, to be submitted.

[8] S. Fan, "Sharp asymmetric line shapes in side-coupled waveguide-cavity systems," Appl. Phys. Lett. 80, 908-910 (2002).

[9] A. E. Miroshnichenko, Yu. Kivshar, C. Etrich, T. Pertsch, R. Iliew, and F. Lederer, "Dynamics and instability of nonlinear Fano resonances in photonic crystals, ” Phys. Rev. A 79, 013809 (2009). 